\title{
Économie solidaire et espace public en Afrique : le cas de la région de Marrakech
}

\author{
Brahim Elmorchid*
}

\begin{abstract}
Résumé
L'interaction entre l'économie solidaire et l'espace public suscite de plus en plus d'intérêt tant par les débats théoriques qu'elle implique que par les réalités socio-économiques qu'elle recouvre. Cette présente contribution ne vise pas à dresser un panorama exhaustif des débats suscités par ce thème. Aussi, après avoir expliqué le rôle capital de l'espace public dans la compréhension de l'économie solidaire, notre propos visera à expliquer les mécanismes par lesquels les activités relevant de l'économie solidaire dynamisent l'espace public. Et à travers une étude de type monographique, nous mettrons à l'épreuve les apports de la littérature se rapportant à cette question.
\end{abstract}

\begin{abstract}
The interaction between the economy of solidarity and public space gives rise to growing interest, both due to the theoretical debates it implies and in light of the socioeconomic realities it involves. The present contribution does not aim to paint a complete picture of the debates on this theme. After explaining the crucial role of public space in any clear understanding of the economy of solidarity, we will focus on explaining the mechanisms whereby the activities pertaining to the economy of solidarity give new impetus to public space. Finally, through a monographic study, we will test the contributions made by the literature relating to this issue.
\end{abstract}

\section{Introduction}

À l'heure de la mondialisation, il est de plus en plus difficile de penser la démocratie sans prendre en compte les interactions qui unissent le politique et l'économique. Et c'est, entre autres, grâce à l'émergence et le

* Département des sciences économiques, Faculté de droit, Université de Marrakech, Marrakech, Maroc. Email : morchid-brahim@yahoo.fr 
développement de l'économie solidaire que ces deux sphères commencent à communiquer. La dimension économique insiste sur la prédominance de l'impulsion " réciprocitaire " dans l'émergence des pratiques et leur consolidation à travers le croisement des ressources. Quant à la dimension politique, elle s'ancre sur cette réciprocité et la construction d'espaces publics autorisant un débat entre les parties prenantes sur les finalités poursuivies et les moyens à mettre en place.

Combinant les activités économiques et le projet politique, l'économie solidaire est devenue une sphère de lecture privilégiée de ce type d'interactions. Elle se présente comme une logique différente à la fois de celle de l'économie de libérale et de celle de l'économie dirigée. Elle se construit sur un mode associatif œuvrant en faveur d'une économie de proximité et d'une démocratie participative. Elle est considérée comme un canal pertinent de la socialisation et de la participation citoyenne aux projets économiques, sociaux et politiques.

Par son refus de toute forme d'exclusion, d'inégalités, de dégradation du patrimoine et des conditions de vie, l'économie solidaire est un engagement politique d'un modèle de développement unique proposé aux citoyens. Elle s'inscrit dans le champ politique comme création d'entité collective dans un contexte souvent conflictuel. L'une des conséquences logiques de ce constat est que l'appréciation de l'économie solidaire passe par la prise en compte de sa dimension politique. Or, la dimension politique de l'économie solidaire ne peut pas être correctement saisie si l'on n'utilise pas un guide théorique puissant, en l'occurrence l'espace public. Ce dernier est devenu le garant de la démocratie participative. Il est à la fois un espace de médiation entre société civile, système étatique et marché, un lieu de légitimation du politique, un espace symbolique permettant de relier entre eux des individus n'appartenant plus à des communautés traditionnelles, et enfin une scène de construction de visions où des acteurs défendant des intérêts différents proposent des versions contradictoires d'un phénomène social donné.

Ainsi, la notion d'espace public est centrale pour la compréhension de l'économie solidaire puisqu'elle permet d'éclairer la constitution du lien social et politique, d'appréhender la construction d'une représentation politique sociale et de comprendre les mécanismes de médiation et d'influence entre les acteurs de la société civile, les élus et les agents économiques.

Par ailleurs, l'espace public peut également subir les effets des activités de l'économie solidaire. En effet, l'économie solidaire entend remédier tout à la fois à la fragmentation du lien social et à la crise de la démocratie représentative. Pour cela, elle favorise le développement d'activités économiques dont la finalité première n'est pas le profit, mais la création de liens sociaux. De plus, elle cherche à générer des dynamiques collectives destinées à faciliter un accès plus large et plus égalitaire à l'espace public. 
Au Maroc, depuis une dizaine d'années, on observe une dynamique des initiatives solidaires avec l'apparition d'organisations qui sont à la fois créatrices de nouveaux liens sociaux, de nouvelles pratiques institutionnelles et de nouveaux rapports économiques. Une problématique d'une importance singulière est qu'il n'existe pas de réflexion suffisante à propos de l'interaction entre l'économie solidaire et l'espace public au Maroc. Et c'est pour contribuer à la relance du débat sur cette question que nous avons choisi d'analyser ce sujet. L'objectif principal est de démonter que l'espace public affecte, mais se trouve aussi affecter par les activités de l'économie solidaire. L'espace public permet de mieux saisir la notion d'économie solidaire. De même,la participation des citoyens à la vie locale peut aider à la reconstruction collective d'un espace public de proximité.

Le présent papier est organisé comme suit. Dans un premier point, nous soulignerons l'importance de l'outil conceptuel « espace public » dans la compréhension de l'économie solidaire. Dans un deuxième point, nous développerons les effets des initiatives de l'économie solidaire sur la reconstruction de l'espace public. Enfin, dans le troisième point nous exposerons les résultats d'une étude de cas relative à l'une des 16 régions marocaines, en l'occurrence Marrakech.

\section{L'espace public : un concept utile pour la compréhension de l'économie solidaire}

\section{L'économie solidaire : un concept multidimensionnel}

Le concept d'économie solidaire a fait l'objet d'un foisonnement de définitions et d'explications. Les termes utilisés et les explications avancées varient en fonction du champ disciplinaire des chercheurs (économistes, anthropologues, sociologues, politologues et chercheurs en communication), mais aussi de la qualité des acteurs impliqués dans cette économie (élus, militants de la société civile, académiciens, ou encore bailleurs de fonds).

L'économie solidaire a été longtemps appréhendée à travers sa dimension socio-économique. Cela se justifie principalement par le fait que l'économie solidaire contribue à découvrir de nouveaux marchés, à créer des emplois, à lutter contre l'exclusion et à réintégrer des personnes en difficulté dans la vie professionnelle. Certaines définitions de l'économie solidaire s'insèrent parfaitement dans cette logique. Ainsi, pour Alcoléa (1999), l'économie solidaire peut être vue comme un secteur spécifique, comme un mode de production, comme une autre manière de penser les relations entre l'économie, le social et la politique ou encore un mode de gestion d'un territoire lié à une politique de développement local. Quant à Demoustier (2001), il définit ce concept comme l'émergence d'activités économiques porteuses de lien social, 
d'insertion et de satisfaction des besoins des groupes populaires marginalisés. Pour Eme et Laville (1995), l'économie solidaire est une économie hybride qui assure le financement de projets sociaux en croisant un financement par le marché, du bénévolat et des aides des collectivités territoriales. Ces projets sociaux prennent des formes multiples (régies de quartier, systèmes d'échanges locaux, jardins de cocagne, etc.) et sont mises en œuvre par des acteurs divers qui, ne limitant pas l'économie à l'échange monétaire, cherchent à dépasser les situations d'exclusion au profit d'un lien de solidarité voulu qui favorise l'invention sociale. Dans le même ordre d'idées, Guigue (2000) considère l'économie solidaire comme une branche qui regroupe des initiatives qui développent des activités et des projets fondés sur des valeurs de partage et de développement durable (finance durable, insertion par l'économique). Elle a une double fonction : produire des biens et des services, et produire des liens sociaux et de la solidarité.

Aujourd'hui, la notion d'économie solidaire ne peut plus être saisie uniquement à travers sa dimension socio-économique. D’abord certains événements et expériences ont été à l'origine d'une forte institutionnalisation de l'économie solidaire. C'est le cas de la création d'organismes publics et/ ou d'un département ministériel dédiés exclusivement aux services de l'économie sociale. C'est le cas également de l'intervention, de plus en plus croissante, des hommes politiques dans le travail associatif, l'émergence des mouvements alter-mondialistes, etc. Ensuite, en s'inspirant de la littérature économique, il semble qu'à l'instar de toute économie, l'économie solidaire a un caractère politique. En effet, il est difficile de penser l'économie en dehors du fait monétaire qui, lui-même, possède une double nature : économique et politique (Cartelier 1996 ; Chaskiel 2000). Et même certains acteurs de l'économie solidaire insistent sur cette dimension politique. L'économie solidaire devient une sorte d'engagement politique par son refus de toute forme d'exclusion, d'inégalités, de dégradation du patrimoine et des conditions de vie, d'un modèle de développement unique imposé à tous les habitants de la planète (Collectif MB2 2001).

Selon Laville (2000), «l'institution d'économie solidaire peut être appréhendée en tant qu'institution intermédiaire dans deux champs qu'elle articule, le champ politique et le champ économique. L'institution d'économie solidaire s'inscrit dans le champ politique comme création d'entité collective dans un espace public conflictuel ». La conséquence logique de ce constat est que la compréhension de l'économie solidaire passe par la prise en compte de sa dimension politique. Or, le recours à la notion d'espace public est capital pour mettre en lumière la dimension politique de l'économie solidaire.

À partir d'engagements citoyens, les activités relevant de l'économie solidaire contribuent à la démocratisation de l'économie. L'économie solidaire 
n'est pas une reproduction de la dérégulation qui tente de remplacer l'action publique par la charité. Elle émane d'actions collectives visant à instaurer des régulations locales, complétant ou suppléant les régulations nationales. Il ne s’agit pas de substituer à la solidarité redistributive une solidarité plus réciprocitaire, mais de définir des modalités de couplage pour compléter la solidarité redistributive par une solidarité réciprocitaire. L'économie solidaire recherche une démocratisation de l'économie en articulant les dimensions réciprocitaires et redistributives de la solidarité afin de renforcer la capacité de résistance de la société à l'atomisation sociale, elle-même accentuée par la monétarisation et la marchandisation de la vie quotidienne (Perret 1999).

L'économie solidaire ne prend sens que par rapport à une économie plurielle, c'est-à-dire une économie ne se réduisant pas à la société de capitaux et au marché, dans laquelle plusieurs logiques économiques peuvent se déployer. Elle met l'accent sur la pluralité des formes de propriété, ce qui revêt une actualité nouvelle à l'heure où les pratiques libérales génèrent des effets de plus en plus perçus comme pervers par l'opinion publique.

L'économie solidaire paraît donc comme une notion multidimensionnelle. D'abord, elle offre une autre manière de pratiquer de l'économie. Les initiatives relevant de l'économie solidaire s'efforcent de subordonner le bien au lien, d'ajuster l'offre à la demande non par les mystères de la main invisible du marché, mais par les mécanismes politiques de la délibération. Ensuite, elle est assimilée à un ensemble d'activités contribuant à la démocratisation de l'économie à partir d'engagements citoyens. C'est un projet politique, celui d'une citoyenneté participative qui, non seulement, prend toute sa place dans la société civile et dans la sphère politique, mais qui a vocation à se développer au sein même de la sphère économique. Enfin, l'économie solidaire paraît comme une utopie. Elle conteste, dans l'ordre symbolique, la pertinence des prétendues lois économiques. Plus encore, elle propose une nouvelle vision de l'ordre social démocratique, fondé non pas sur la maximisation individuelle de l'utilité, mais sur la recherche collective de solidarités démocratiques. En ce sens, elle constitue une source d'espoir pour les générations futures.

\section{Le rôle de l'espace public dans la régulation de l'économique}

L'espace public est avant tout un espace de paroles et de débat. Dans le cas des activités de l'économie solidaires, il y a l'idée de la construction des besoins sociaux. L'organisation de la production et de la distribution, de même que l'ajustement des prix et des quantités passent souvent par une forme de délibération entre acteurs (Fraise 2003). Ils n’obéissent ni aux règles de l'économie marchande, ni aux règles de l'économie dirigée. En d'autres termes, les variables économiques, en particulier les prix, ne sont 
plus considérées comme exogènes, imposées aux acteurs économiques. Elles sont davantage soumises aux délibérations et à la discussion entre les acteurs des activités relevant de l'économie solidaire. Plusieurs exemples permettent d'illustrer ces propos. Prenons d'abord la finance solidaire. En pleine expansion dans les pays africains, cette activité obéit à une médiation directe. Les porteurs de projets sont souvent accompagnés et orientés. De même, dans le commerce équitable, l'objectif est la recherche d'un juste tarif lors des échanges entre les producteurs du Sud et consommateurs du Nord. Enfin, dans les services publics de proximité (eau, électricité, route, etc.), la construction conjointe de l'offre et de la demande joue un rôle central dans la socialisation des besoins, dans la détermination du contenu des services, ainsi que dans la mobilisation des différentes ressources (marchande, non marchande et non monétaire) nécessaires à la pérennisation de l'activité.

S'inspirant des travaux d'Habermas (1997), Fraisse (2003) et Dacheux (2000), il est possible de montrer que l'espace public est nécessaire pour la compréhension des activités relevant de l'économie solidaire. Comme le montre Habermas dans « Droit et démocratie », la médiation touche à la fois le domaine économique et politique. L'espace public est l'instance de médiation entre la société civile, le système étatique et le système économique. D’une manière plus large, l'espace public assure la médiation entre la sphère privée et la sphère publique. Les activités de l'économie solidaire permettent une socialisation et une publicisation de besoins (aides aux personnes démunies, aide aux personnes âgées...) qui étaient, et en grande partie demeurent, pris en charge dans le cadre de la famille ou du voisinage, voire de l'économie informelle (Caillé 2001).

L'espace public autorise également la confrontation et la négociation entre les différentes parties prenantes d'une activité socio-économique, en particulier les usagers, les pouvoirs publics, les bénévoles et les professionnels. C’est la communication qui permet de surmonter les difficultés liées à la divergence d'opinions et faire prévaloir l'intérêt général de la communauté, caractéristique essentielle de l'économie solidaire. Pour que l'espace public soit celui de l'exercice d'une réflexion critique qui renforce la société démocratique et non un espace d'affrontement désintégrant cette société, il faut que les acteurs de cette société partagent un même espace communicationnel.

Par ailleurs, l'espace public est une méthode de construction des activités solidaires. Les promoteurs de ce type d'activités saisissent souvent les réunions publiques formelles ou informelles pour présenter leurs projets, leurs réussites, mais aussi les difficultés rencontrées. Ce type d'initiatives présente deux avantages. D’abord, il constitue une étape méthodologique à part entière dans la construction des activités solidaires. Ensuite, lorsqu'elle est organisée, la mise en débat public permet à ceux dont la parole est 
généralement disqualifiée par les institutions et les experts à la fois un apprentissage de la prise de parole, une valorisation externe de leur démarche, une interpellation sur les obstacles rencontrés dans la création d'activités, une appropriation de leur environnement institutionnel, et enfin la rencontre et la confrontation avec d'autres porteurs de projet. Plus généralement, le repérage des besoins et la structuration de l'offre ne passent pas forcément par le recours aux outils classiques de la création d'entreprise (étude de marché, étude de faisabilité, étude marketing...), mais mobilisent également des ressources militantes et volontaires pour faire connaître les produits et services auprès du public.

Une autre manière de saisir l'influence de l'espace public sur les activités de l'économie solidaire consiste à le considérer comme un lieu de régulation sectoriel et de reconnaissance de la pluralité des logiques économiques sur un territoire (Fraisse 2003). En effet, afin de créer et pérenniser des espaces de délibération communs dans une organisation productive, il est nécessaire d'agir sur les régulations économiques sectorielles ou territoriales. Parce qu'elles se heurtent aux insuffisances des institutions légitimes de production de richesse et de solidarité (entreprises privées et publiques), les initiatives d'économie solidaire mettent en œuvre des projets socio-économiques qui interrogent les politiques publiques " historiquement construites sur le cloisonnement entre économies marchande et non marchande » (Laville et Nyssens 2001). Vu le caractère autonome des organisations exerçant les activités solidaires, l'espace public peut alors être le moyen d'une interpellation et de négociation avec les pouvoirs locaux et les autres acteurs économiques d'un mode de régulation locale qui reconnaît la place et la spécificité des initiatives de l'économie solidaire.

L'espace public est considéré aussi comme un lieu de contrôle et de légitimation du politique. Il est le garant de la démocratie. C'est par l'espace public que les citoyens ont accès aux informations politiques, qu'ils peuvent débattre et se forger une opinion et qu'ils peuvent choisir les personnes qui exerceront le pouvoir politique. C'est par l'espace public que les citoyens se sentent non seulement consommateur de droit, mais aussi auteur de ce droit.

Remarquons enfin que l'espace public permet de relier trois espaces (domestique, public et politique). Cette liaison est assurée essentiellement au moyen de l'espace médiatique, mais aussi par les champs politiques qui les connectent deux à deux : les espaces de médiation institutionnelle et les espaces civils. Comme l'indiquait Habermas dans "L'espace public », l'opinion que chacun peut se forger des problèmes de la société se forge aussi dans l'espace domestique via les mass media (Habermas 1978). Vu sous cet angle, l'espace domestique constitue le fondement de l'espace public. 


\section{Le rôle de l'économie solidaire dans le renouvellement de l'espace public}

\section{Vulgarisation des concepts économiques}

Avec le développement des activités de l'économie solidaire comme thème de débat, l'espace public s'est enrichi sur le plan terminologique. Initialement appropriés par les économistes, beaucoup de concepts de la micro et de la macro économie ont été popularisés et vulgarisés. Des termes comme épargne, micro finance, circuit économique, profit, offre, demande, sont venus enrichir le vocabulaire employé lors des débats, négociations et confrontations au sein de l'espace public. Plus encore, ces nouveaux termes ont été vulgarisés de façon pragmatique grâce à leurs utilisations fréquentes et les efforts d'explication et de communication déployés à la fois par les professionnels et les simples citoyens.

\section{Développement de la communication solidaire}

L'économie solidaire est, tout à la fois, un ensemble d'initiatives économiques cherchant à tisser des liens sociaux, un projet utopique et une notion scientifique. Une telle polysémie nécessite naturellement une bonne communication.

Avec l'émergence de l'économie solidaire, l'audience d'économie responsable, plurielle et solidaire a été progressivement installée et élargie. Les militants et les réseaux civiques sont devenus les vecteurs de diffusion de l'économie solidaire. De même, des médias alternatifs et des médias sociaux (presse interne des associations et des organisations de l'économie sociale) commencent à faire leur apparition. Enfin, on assiste de plus en plus à la diffusion et à la pérennisation des médias spécialisés dans l'économie solidaire (journaux, sites Internet, etc.) et l'alimentation des rubriques « économie solidaire » des médias généralistes.

Une bonne communication installée sur toute la chaîne qui va de la production à la consommation (ou usage) du produit ou service est la pierre angulaire du développement d'une vision et d'une stratégie globale pour une économie responsable, plurielle et solidaire. Elle constitue un préalable à la création de projets et d'outils transversaux impliquant toutes les parties prenantes.

Certains auteurs (Crépy 2006 et Dacheux 2006) soulignent les difficultés que rencontre la communication de l'économie solidaire dans l'espace public. Deux catégories de difficultés ont été identifiées. La première a trait à la faible visibilité médiatique de l'économie solidaire. En raison de l'absence de formation spécialisée, les journalistes ont souvent tendance à se focaliser sur l'activité que sur le concept dans lequel s'inscrit cette activité. Pour beaucoup de journalistes, les différentes initiatives de l'économie solidaire 
sont perçues comme relevant de la microéconomie. À ce déficit de communication s'ajoute souvent l'absence de statistiques fiables sur les activités de l'économie solidaire, en particulier dans les pays en développement. La seconde difficulté de communication rencontrée par l'économie solidaire est liée aux contours de l'espace public. Celui-ci est un espace de confrontation politique dominé par une idéologie hégémonique. Or le projet porté par l'économie solidaire va à l'encontre de cette vision. Dans ces conditions, l'économie solidaire est condamnée à subir les représentations dominantes.

\section{Enrichissement du débat public}

Comme nous l'avons déjà souligné, l'économie solidaire se démarque à la fois de l'économie marchande libérale et de l'économie dirigée. À la différence de l'économie libérale, elle ne repose pas sur la recherche de profits, mais sur la volonté de trouver l'échange le plus équitable possible de biens, de services ou de relations. Et à la différence de l'économie dirigée, elle ne s'insère pas dans le processus de régulation étatique ou bureaucratique. Au lieu de s'inspirer des règles édictées par le haut, l'économie solidaire se construit sur un mode associatif dont la régulation se ferait plutôt par le bas (Fraisse 2003). Elle repose sur la proximité comme logique d'action collective et comme démarche de travail.

L'une des principales caractéristiques des activités relevant de l'économie solidaire est la mise en place des « espaces publics de proximité » (Laville 1994), c'est-à-dire des espaces d'argumentation et de paroles communs permettant aux différentes parties prenantes (usagers, professionnels, bénévoles, pouvoirs publics, etc.) de co-construire l'offre et la demande, de co-organiser la production et de co-décider des prix et des quantités. Et dès lors qu'il y a constitution d'espaces de débats et de co-construction des choix ou bien encore d'intermédiation, la question de l'espace public est ouverte. Dans le cas d'associations de développement local par exemple, les adhérents discutent des projets à entreprendre, décident du niveau et des sources de financement, fixent les procédures et les modalités d'accompagnement de chaque projet et enfin procèdent à l'évaluation des réalisations. De même, dans une association de quartier, ce sont les discussions entre les dirigeants, les habitants et les pouvoirs publics qui fixent les tâches prioritaires à accomplir et le prix des prestations.

Ces espaces publics de proximité deviennent ainsi des espaces de négociation démocratique d'activités socio-économiques. Ils permettent, comme le signale Fraisse (2003), de publiciser certains besoins sociaux autrefois pris en charge dans le cadre domestique (aide aux personnes âgées par exemple) ou par les collectivités locales (eau potable, électricité, routes, 
etc.). Ils contribuent à jeter un pont entre la sphère privée et la sphère publique ou à élargir cette dernière. Ils permettent aussi de faire surgir de nouvelles revendications sociales et à attirer l'attention des pouvoirs publics sur les problèmes qui nécessitent un traitement urgent.

Par ailleurs, le suivi du processus de mise en œuvre de nouvelles activités relevant de l'économie solidaire présente la caractéristique de proposer un cadre collectif (Codello-Guijarro 2003). Un tel cadre représente une grille intermédiaire entre l'espace privé et l'espace domestique qui permet de reprendre confiance en soi, de percevoir la dimension politique de l'exclusion, d'échanger des bonnes pratiques, de se soutenir moralement et, souvent, de définir un certain nombre de revendications collectives, de prendre part au débat public local (Guérin 2003). En d'autres termes, l'économie solidaire met en place des espaces de médiation propres à la société civile qui permettent à des exclus d'accéder à nouveau à l'espace public (Fraisse 2003). À cela, il convient d'ajouter le développement des réseaux thématiques dédiés à l'économie solidaire. À travers le débat créé et l'échange d'expériences, ces réseaux permettent l'élargissement du champ d'action de l'économie solidaire. Ils créent également une sorte de synergie entre les actions des militants. Ils permettent enfin de faire la promotion de l'économie solidaire dans l'espace public et tentent de peser sur les politiques publiques.

Il paraît ainsi que l'économie solidaire est utile pour dynamiser l'espace public. Elle constitue une réponse politique pragmatique aux maux dont souffre cet espace. Elle contribue à pluraliser un espace médiatique qui se concentre, à rendre visible une part du social qui n'intéresse plus guère les entreprises médiatiques. De plus, elle combat le repli sur la sphère privée en vitalisant l'espace public local et en créant des espaces de médiation permettant à des exclus de se constituer en collectif pouvant défendre leur point de vue dans l'espace public. Enfin, elle participe au renouveau de l'engagement citoyen en conjuguant discours critique contre le capitalisme et action sociale concrète. Mais l'économie solidaire n'est pas seulement une réponse pragmatique à la crise politique, c'est aussi un projet utopique pouvant remédier au déficit symbolique qui affecte les démocraties.

Signalons aussi que la recherche de la proximité des relations d'échange est une caractéristique profonde de ces expériences. Habermas a remarqué combien l'éloignement spatial et vécu entre les usagers et la bureaucratie des services publics était une des raisons fortes de l'instrumentalisation de la communication. Le "médium du pouvoir " condense la relation communicationnelle du monde vécu en règles impersonnelles et aveugles du sous-système politique. En conséquence, l'espace public est déconnecté de la vie réelle et le débat est remplacé par les dispositifs réglementaires. Il semblerait que les politiques de « décentralisation » restent un vain mot si 
elles ne sont pas relayées par un immense déplacement vers le terrain concret des rapports avec les usagers et les citoyens.

\section{Interactions entre économie solidaire et espace public dans la région de Marrakech}

Depuis le début des années quatre-vingt-dix, les initiatives se revendiquant de l'économie solidaire se sont multipliées dans toutes les régions du Maroc. Elles recouvrent essentiellement la création de nouveaux services ou l'adaptation de services existants (services de la vie quotidienne, d'amélioration du cadre de vie et de protection de l'environnement) et l'intégration dans l'économie des populations défavorisées à travers les activités génératrices de revenus.

Pour appréhender l'interaction entre les activités relevant de l'économie solidaire et l'espace public au Maroc, nous avons mené une enquête qualitative (interviews) auprès de 15 associations et coopératives actives dans la région de Marrakech. Le choix de cette région a été motivé par deux considérations : l'une historique et l'autre économique. En effet, l'espace public actuel de Marrakech est le résultat de plusieurs siècles de mutations sociales. De plus, la région de Marrakech occupe aujourd'hui une place de premier plan en matière de développement du mouvement associatif au Maroc. Nous présentons dans ce qui suit les principaux résultats de cette enquête.

\section{Quelques caractéristiques socio-économiques de la région de Marrakech}

Connue administrativement sous le nom « Marrakech-Tensift-El Haouz », la région de Marrakech est l'une des seize régions du Maroc. Elle se trouve au centre du pays, et englobe une partie du haut Atlas. Sa superficie est de $31160 \mathrm{~km}^{2}$, ce qui représente 4,5 pour cent du territoire national, pour une population de plus trois millions d'habitants.

La population de la région dans son ensemble est relativement jeune: plus de 35 pour cent a moins de 15 ans. La population en âge d'activité représente plus de 50 pour cent, ce qui indique qu'une personne sur deux est potentiellement active. Quant à la population du 3e âge, elle ne dépasse pas 10 pour cent.

Le taux d'activité dans la région s'élève à 34 pour cent. La répartition de la population active par grands secteurs donne 52 pour cent au primaire, 18 pour cent au secondaire et 30 pour cent pour les services.

La région de Marrakech-Tensift-Al Haouz est globalement pauvre comparativement aux autres régions du royaume. De même, le taux d'analphabétisme est l'un des plus élevés au Maroc. Il dépasse 50 pour cent pour l'ensemble de la région et 65 pour cent au niveau du monde rural. 
L'économie de la région repose principalement sur l'agriculture et l'élevage, le tourisme, l'industrie de transformation, l'artisanat et dans une moindre mesure sur la pêche. L'agriculture représente le secteur clef de l'économie régionale. Elle occupe plus de 50 pour cent de la population active. Quant à l'industrie, elle connaît un essor assez important. Elle est constituée essentiellement de l'agro-industrie, de la chimie et de la parachimie.

Le tourisme est le second secteur important dans la région. La capacité d'hébergement représente plus de 25 pour cent du total national. Ce secteur a connu ces dernières années une progression notable. On assiste à un développement rapide de l'infrastructure hôtelière, particulièrement dans la ville de Marrakech. En relation avec ce secteur, l'artisanat a enregistré des résultats satisfaisants. Enfin, la région ne dispose que d'un seul port de pêche à Essaouira. Mais son activité reste très limitée comparativement aux autres ports de pêche situés au sud du pays.

\section{Conditions d'émergence de l'économie solidaire}

Le Maroc est parmi les pays africains leaders en matière de développement des activités relevant de l'économie solidaire. Il compte plus 40000 associations caritatives et de développement local. L'essor de l'économie solidaire et de l'économie sociale en général trouve son origine dans la crise qui caractérise la société marocaine. Cette crise n'est pas seulement d'ordre économique, mais aussi politique.

En premier lieu, l'émergence de l'économie solidaire constitue une réaction à la crise économique qui a secoué le Maroc au début des années quatre-vingts et la mise en œuvre du programme d'ajustement structurel (PAS) sous l'égide et les recommandations du Fonds Monétaire International et de la Banque Mondiale. Pour des raisons d'équilibres macroéconomiques, l'État s'est progressivement retiré des secteurs sociaux en appliquant une politique d'austérité budgétaire. Cela a donné lieu à un accroissement des déséquilibres sociaux, en particulier dans le monde rural. Ces déséquilibres se sont aggravés suite à la succession de plusieurs années de sécheresse. Le taux de pauvreté n’a cessé de croître. Au début du XXIe siècle, presque un marocain sur cinq vit au dessous du seuil de pauvreté (Direction de la Statistique 2001). Et ce sont principalement les victimes de cette nouvelle situation qui ont été les artisans du développement des activités de l'économie solidaire.

Délaissé par l’État et encadré en partie par les membres de la classe intellectuelle, les populations démunies décident de se prendre en charge. Il s'agit là d'une sorte de combat contre la domination d'ordre économique, symbolisée par l'avènement de la société de marché. Ainsi, en proposant différents services aux personnes démunies et non solvables (transports, 
services de base, etc.), l'économie solidaire enrichit la palette d'action des pouvoirs publics. En plaçant au cœur de la lutte contre l'exclusion, la notion de réciprocité, l'économie solidaire contribue à restaurer cet horizon d'égalité entre les citoyens qui fait défaut aux systèmes démocratiques jeunes. De même, face à l'application du modèle néolibéral qualifié par certains de « sauvage », l'économie solidaire propose un autre modèle de développement économique basé sur la restauration du lien social par la mise en place d’espace public de proximité pour développer des activités économiques répondant aux besoins des citoyens et respectant l'environnement.

En deuxième lieu, l'essor de l'économie solidaire constitue une réponse à la crise politique. Depuis l'indépendance du Maroc, ce sont essentiellement les citoyens électeurs d’en bas qui ont contribué aux « succès » des échéances électorales. Ces échéances ont toujours été pour eux un espoir de voir les choses changer. Mais, après plusieurs années d’implication, ces citoyens sont déçus, instrumentalisés. Ils se sont alors retirés de ce jeu politique. Ils ont également perdu confiance en les partis politiques. En effet, les partis politiques marocains sont des structures construites autour de l'ambition personnelle du chef (secrétaire général), qui est souvent leur principal bailleur de fonds. L'adhésion à ces partis est très faible et elle a un caractère circonstanciel (période des élections). Les conflits internes sont toujours résolus par la scission. Lors des rares congrès des partis politiques, les mécontents finissent toujours par abandonner le parti pour en créer un nouveau, ou rejoindre un autre déjà existant. Depuis 1996, tous les grands partis marocains ont été gravement affaiblis par les sécessions et les divisions. Et tandis que de nouveaux partis voient le jour chaque année, aucun ne se dissout.

Par ailleurs, les députés ne sont jamais élus sur la base d'un programme, mais plutôt sur la base de relations personnelles et parfois professionnelles. Comme l’a bien souligné Schmitter (1999), dans les « néo-démocraties », les personnalités priment sur les partis. Il s’agit bien plus pour des hommes importants de tenter de protéger leur statut en s'affiliant à un parti ou en créant un, que pour des politiciens d'atteindre une envergure nationale en faisant carrière dans un parti. Que la plupart des partis n’aient ni programme politique, ni capacités d'analyse politique n’est donc pas surprenant. La très faible participation aux dernières échéances législatives organisées au Maroc en septembre 2007 témoigne parfaitement de cette crise politique.

Parallèlement, la presse écrite partisane (journaux des partis politiques) a accusé un recul significatif aussi bien sur le plan quantitatif (nombre d'exemplaires vendus par jour) que qualitatif (qualité des articles publiés). Elle a été délaissée à la fois par les lecteurs et par les journalistes qui ont préféré la presse indépendante, jugée plus crédible et plus transparente. 
La perte de confiance en les acteurs du système politique (à part l'institution royale) a précipité l'apparition d'associations ayant comme principal but l'encadrement des citoyens sur le plan social, économique et même politique. Relevant de l'économie solidaire, ces associations ont été à l'origine de la mise en place d'espaces publics de proximité.

Caractéristiques de l'économie solidaire dans la région de Marrakech A partir des interviews effectuées et l'observation non participante, nous avons relevé les principales caractéristiques de l'économie solidaire dans la région de Marrakech. Il convient d'abord de préciser que les fondements et les pratiques de l'économie solidaire sont très anciens dans la région. En effet, la twiza (ou la collectivité au service du particulier) est une pratique associative très ancienne. La twiza est un acte de société qui vise à réaliser une œuvre de bienfaisance en faveur de la collectivité ou des particuliers, généralement nécessiteux. C'est un comportement spontané dont l'objectif est d'unifier les forces humaines (et les moyens parfois) pour un développement équitable de la localité ou apporter l'aide à ces nécessiteux de la localité. Ainsi, une famille peut demander, grâce à la coutume twiza, la participation des villageois pour une tâche importante, à la simple condition (dans la mesure du possible) de nourrir les participants. De plus, la twiza a toujours été considérée comme une véritable fête.

L'économie solidaire dans la région de Marrakech se présente essentiellement sous forme associative et dans une moindre mesure sous forme coopérative. Ce sont les deux seuls statuts juridiques que peuvent prendre les entreprises opérant dans l'économie solidaire.Ces organisations assurent leur financement de façon hybride. Outre la contribution des membres, les fonds proviennent des recettes marchandes, des transferts publics et parfois même des aides internationales.

La majorité des activités relevant de l'économie solidaire sont insérées dans des services publics (desserte en eau potable, scolarisation des enfants, accès au réseau d'électricité, construction de routes, etc.), l'agriculture, l'artisanat et rarement la protection de l'environnement. Conformément aux développements conceptuels, les activités de l'économie solidaire articulent des principes qui tiennent à la fois de la réciprocité, de la redistribution et du marché, et même parfois des relations domestiques. Elle peut porter sur la solidarité entre les territoires et au sein de ceux-ci, le partage des risques et des richesses entre groupes sociaux, la solidarité entre générations présentes et la solidarité avec les générations futures dans la perspective d'un développement durable. À l'évidence, vu les caractéristiques territoriales de la région, le nombre d'associations et coopératives opérant dans l'économie solidaire est relativement supérieur dans le milieu rural (environ 2000 sur 
3000 que compte la région). De même, la majorité de la population qui a besoin de l'économie solidaire est issue du secteur informel. Ce secteur est fortement enraciné dans l'histoire de la réalité marocaine, surtout par ses effets d'exclusion de la main-d'œuvre nationale au marché du travail formel.

En ce qui concerne la participation selon le genre dans les associations et coopératives développant les activités de l'économie solidaire, nous remarquons que les hommes sont majoritaires. Le contexte culturel marocain est encore conservateur. La vie publique et la participation politique ont toujours été vues comme un espace où les hommes se sentent plus à l'aise. L’histoire du Maroc est marquée par des inégalités entre hommes et femmes. Toutefois, ce sont les associations et coopératives dominées par les femmes qui réussissent mieux dans leurs missions. Ces associations opèrent généralement dans l'éducation non formelle et les travaux d'artisanat.

Nous remarquons également l'absence de coordination entre les différentes associations quant aux domaines d'intervention et aux actions entreprises. Au lieu de renforcer et améliorer l'efficacité et la performance des associations déjà existantes, on assiste à leur fractionnement et leur multiplication. Le principal motif de cette situation reste les conflits entre les membres. En effet, malgré l'apport indéniable des associations en matière de développement local, elles sont devenues source de rente. Beaucoup d'associations sont en quête de contrats auprès d'agences de développement, d'ONG internationales ou même d'entreprises étrangères implantées au Maroc. Elles mettent en œuvre uniquement les projets de développement pour lesquels elles trouvent un financement. Souvent, leurs leaders combinent les fonctions de consultants publics ou privés et de " courtiers en développement ». Le travail associatif devient producteur de profit illégitime qu'on peut qualifier de « rente associative » (Elmorchid 2007). Par ailleurs, en raison de la perte de confiance des citoyens en les partis politiques, certains politiciens n'hésitent pas à adhérer ou même à créer leurs propres ONG. En quête de légitimité, ces politiciens préfèrent désormais utiliser l'étiquette associative afin de se démarquer des partis politiques et de leur mauvaise réputation. Aujourd'hui, beaucoup de leaders d'ONG de développement ont de multiples facettes et se situent entre les deux mondes de la politique et de l'associatif. Par conséquent, la distinction entre société civile, État et marché, sur laquelle se fonde une bonne partie du discours actuel sur le développement commence à se décomposer.

Enfin, la communication des associations qui opèrent dans l'économie solidaire est dans un état embryonnaire. La presse spécialisée est absente et seuls quelques journaux consacrent de temps en temps un espace réduit à la publication d'annonces et d'articles associatifs. Les associations reposent encore sur un militantisme de voisinage et sur une communication diffusée dans l'espace privé, au cours de réunions au domicile ou au cours d'activités 
de voisinage. Cela explique, entre autres, la difficulté des associations à acquérir une véritable visibilité dans l'espace public. Relativement peu d'associations parviennent à une place nationale dans l'espace politique, et souvent, il s'agit d'associations dont les militants sont aussi acteurs de partis politiques ou sont reconnus, à titre personnel, dans l'espace public, en tant qu'experts. La voix des associations est loin d'être institutionnelle. Elle ne s'adresse à l'espace public qu'en raison de la qualité personnelle de ceux qui s'expriment.

La communication interne des associations est dominée par les relations personnelles entre militants, c'est-à-dire le réseau de relations particulières qui structurent la sociabilité associative. Les questions de personne, voire les susceptibilités individuelles, jouent un grand rôle dans les associations. C'est aussi ce qui explique la fragilité institutionnelle des associations, souvent déstabilisées par le départ d'une personnalité ou l'arrivée d'une autre.

\section{La nouvelle physionomie de l'espace public}

Après plusieurs années de pratiques d'activités solidaires dans la région de Marrakech, l'espace public a connu un élargissement et un certain dynamisme. L'enquête effectuée nous a permis de constater que l'espace public local n'est pas seulement un espace de co-construction de l'offre et de la demande, un espace de débats publics locaux et un espace de médiation. Il est devenu également un espace de lutte contre l'exclusion, un espace de reconnaissance et un espace plus ouvert aux femmes.

D'abord, conformément aux développements théoriques, l'espace public de la région est un espace de co-construction de l'offre et de la demande. Ainsi, à l'inverse des activités économiques marchandes, les pratiques de l'économie solidaire sont mises en place dans un cadre collectif. La logique du producteur à la recherche du profit face aux consommateurs à la recherche d'un maximum de satisfaction est remplacée par un contrat collectif permettant aux différentes parties de construire un produit ou un service le plus satisfaisant possible (maximisation de l'utilité sociale). Dans le cas des associations de développement local par exemple, l'intérêt individuel est noyé dans l'intérêt de la collectivité. De même, dans le cas des associations de quartiers, tout le monde est mobilisé pour offrir des services à moindres coûts. Au bout du compte, tout le monde est gagnant : les citoyens du quartier qui ont la responsabilité de la gestion de l'association, les habitants qui ont certains besoins et payent un service, les pouvoirs publics qui subventionnent une partie du fonctionnement, les professionnels qui encadrent les usages, etc.

Ensuite, l'espace public, objet de l'étude, est un espace de médiation entre les acteurs économiques, les citoyens et les services publics. Ainsi, face au retrait de l'État de la plupart des services sociaux, conjugué à la carence des services privés, les citoyens prennent l'initiative de se prendre 
en charge à travers les services solidaires. Le partenariat est le système le plus souvent utilisé. Pour une bonne réussite des projets solidaires, et sous l'égide des autorités publiques, les acteurs privés, les collectivités locales et les militants des activités solidaires participent au débat sur les projets à développer, les mécanismes de mise en œuvre, les modalités de financement et le suivi des réalisations. Bref, à travers des rencontres et des débats fructueux, tout le monde œuvre pour le bien être du territoire. D'une logique du changement par le conflit, on est passé à une logique de changement par la pacification des échanges entre les acteurs. L'idée est de construire des orientations des politiques à partir de la multiplicité des acteurs locaux, quelles que soient leurs origines et leurs tendances. Les sensibilités politiques et ethniques sont souvent mises à l'écart. Plusieurs exemples témoignent de cette collaboration et de cette médiation dans l'espace public local. C'est le cas d'une partie du réseau routier dans le monde rural qui a vu le jour grâce au partenariat fructueux entre le Ministère de l'équipement, les collectivités locales et les associations. C'est le cas également de l'électrification rurale qui a enregistré un grand succès grâce au PERG (Programme d'Electrification Rurale Global). Il s'agit d'un programme mis en place par les services concernés (Office national d'électricité) en partenariat avec les collectivités locales et les associations de développement local.

L'espace public de la région de Marrakech est un espace de débat, de concertation où tombent souvent les préjugés. En effet, l'économie solidaire est une démarche collective de développement local durable et responsable. Elle met en place des espaces de concertation, des cellules de réflexion, des comités de coordination, des comités de pilotage où les différents acteurs sont appelés à se parler, à discuter en toute sincérité. Mais, cela n'est pas toujours chose aisée. Les débats sont parfois source de colère et de mécontentement. Et c'est grâce à ce type de réactions que les projets se construisent solidement et que les préjugés tombent. Dans les zones rurales de la région par exemple, les citoyens avaient toujours une sorte de méfiance et de réticence à l'égard de l'emprunt, mais après plusieurs réunions avec les professionnels du micro crédit, ils ont finalement accepté ce mode de financement, malgré les taux d'intérêt relativement élevés. Certains d'entre eux sont même allés plus loin en optant pour l'ouverture de comptes, soit auprès de la Poste, soit auprès des banques, qui commencent d'ailleurs à installer leurs agences dans les zones relevant du monde rural. Un deuxième exemple concerne le programme d'alphabétisation des adultes. Au début, et pour des raisons essentiellement culturelles, ce programme n'a pas eu le succès escompté. Mais après plusieurs campagnes de sensibilisation et d'explications, les concernés (essentiellement les femmes) ont accepté d'adhérer massivement au programme en question. 
Les activités relevant de l'économie solidaire offrent un espace de citoyenneté, un espace de valorisation des individus et un espace de réintégration. En effet, à travers les rencontres et les débats, le travail associatif a permis à beaucoup d'individus de retrouver confiance en soi et en la société. Il leur a permis également de gagner la reconnaissance des autres. Les diplômés chômeurs par exemple se sentaient complètement exclus de la société. La multiplication des grèves de la faim témoigne de leur désespoir. Mais à travers les initiatives de l'économie solidaire, en particulier le montage de petits projets financés et suivis par les associations, ils ont réintégré la société. Et avec leurs revenus, certains d'entre eux ont fondé un foyer et commencé à faire profiter les autres de leurs expériences. Un autre exemple frappant est celui des handicapés. Avant, ils étaient abandonnés. Seules leurs familles leur venaient en aide. Mais aujourd'hui, les choses ont changé. Avec la multiplication des associations qui œuvrent pour l'amélioration des conditions de vie des handicapés, plusieurs projets ont vu le jour. C'est le cas des centres de rééducation physique, des maisons d'handicapés et des microprojets conçus particulièrement pour cette catégorie de citoyens.

Avec ce type d'initiatives, l'économie solidaire ne remplit pas uniquement la fonction de solidarité, mais surtout une fonction d'intégration et de réintégration sociale. Elle permet aux individus de se reconnaître en tant qu'habitants et en tant que citoyens et par la même peut les conduire à se faire reconnaître par l'administration et le pouvoir politique (Laville 1999).

Enfin, avec le développement des activités de l'économie solidaire, l'espace public dans la région de Marrakech est devenu plus ouvert aux femmes. L'approche genre commence à marquer l'espace public. Avant, les femmes étaient enfermées dans leur espace privé (espace domestique). Mais l'émergence des initiatives relevant de l'économie solidaire leur a permis d'intégrer l'espace public en participant aux réunions publiques, en devenant animatrices locales, en créant des activités génératrices de revenus et en développant des réseaux de coopération. La conséquence en est la naissance d'un cadre de communication et de sociabilité pour les femmes, en particulier dans le monde rural. Traditionnellement réservés aux hommes, des thèmes comme les droits de l'Homme et la participation politique, commencent à faire l'objet de débat féminin.

\section{Conclusion}

Au terme de cette analyse qui n'est que l'amorce d'une réflexion en cours de construction, on remarque que l'espace public et l'économie solidaire sont interdépendants. D'une part, le concept d'espace public est primordial pour la compréhension de l'économie solidaire. Comme nous l'avons souligné dans les développements précédents, l'espace public permet d'éclairer la 
constitution du lien social et politique, d'appréhender la construction d'une représentation politique d'un fait social (ici, l'économie solidaire) et de comprendre les mécanismes de médiation et d'influence entre les acteurs de la société civile, les élus et les agents économiques. L'espace public offre une modélisation susceptible de mieux saisir les caractéristiques de l'économie solidaire.

D'autre part, l'essor de l'économie solidaire donne lieu à un renouvellement de l'espace public en montrant comment se constituent de nouveaux lieux de médiation entre les sphères économiques, sociales et politiques. De même, l'économie solidaire souligne une dimension souvent ignorée dans l'espace public : la dimension économique. En effet, outre la dimension politique et psychosociale, l'espace public possède une dimension économique puisque les initiatives solidaires permettent de penser l'espace public comme étant un espace de co-construction du développement local. L'économie solidaire permet de remédier partiellement à la décomposition du lien social et à la crise que traverse la société. Aussi, elle favorise le développement d'activités économiques dont la finalité première n'est pas le profit, mais la création de lien social et ce, à travers diverses voies imbriquées : l'ouverture de la plupart des services publics à l'implication des usagers, l'encadrement des jeunes, la réhabilitation des citoyens souffrant de l'exclusion, le renforcement de la citoyenneté, la préservation de l'environnement, la lutte contre l'analphabétisme, etc. De plus, l'économie solidaire génère des dynamiques collectives destinées à faciliter un accès plus égalitaire à l'espace public.

L’observation de la réalité du terrain (cas de la région de Marrakech) nous a permis de constater que l'émergence des activités de l'économie solidaire constitue une réaction à la double crise que traverse la société marocaine : la crise économique et la crise politique. Ces activités ont considérablement dynamisé l'espace public de la région. Elles ont été à l'origine d'un débat public entre toutes les composantes de la société. Le but était de développer un entrepreneuriat collectif guidé par une logique de développement territorial. Cette dernière n'étant ni monolithique ni confinée aux sphères administratives, mais résultant d'un compromis public entre trois logiques d'actions différentes : celle des acteurs économiques, celle des institutions et celle des citoyens.

\section{Références}

Alcoléa, A.M., 1999, « De l’économie solidaire à l'économie solidaire territoriale », La Varenne communication à la journée d'études « Les autres figures de l'économie ».

Archambault, A., 1995, « L’économie alternative, forme radicale de l’économie sociale », RECMA, n²56.

Caillé, Á., 2001, « La société civile mondiale qui vient », in Laville et al., Association, démocratie et société civile, Paris : La Découverte, Mauss, Crida,pp. 188-190. 
Cartelier, J., 2001, La monnaie, Paris : Flammarion.

Chaskiel, P., 2000, « L'étonnante neutralisation de la monnaie », Sciences de la société, n50/51.

Codello-Guijarro, P., 2003, « Vers la construction d'un espace public de proximité », Hermès, n³6, pp. 83-90

Collectif MB2, 2001, Pour une économie alternative et solidaire, Paris : L'Harmattan.

Dacheux, E., Goujon, D., 2000, « L'économie solidaire à l'aube du troisième millénaire », Organisations et territoires, vol. 9, n² 2.

Dacheux, E., Goujon, D., 2002, « De nouveaux outils pour comprendre l’économie solidaire », RECMA, no. 284.

Dacheux, E., 2003, «Un nouveau regard sur l'espace public et la crise démocratique », Hermès 36, pp. 195-204

Dacheux, E., Goujon, D., 2001, « Comprendre l’économie solidaire », Actes du colloque CNRIUT, St Etienne : Presses Universitaires de Saint-Étienne.

Demoustier, D., 2001, L'économie sociale et solidaire, Paris : Syros.

Eme, Â. et Laville, J.-L. 1999, (dir.), Cohésion sociale et emploi, Paris, Desclée de Brouwer.

Eme, B. et Laville, J-L., 1995, « Économie plurielle, économie solidaire », La Revue du Mauss, $n^{\circ} 4$.

Floris, B., 2003, « Espaces publics de proximité », Revue Hermès, n³6, pp. 129-135.

Fraisse, L., 2003, «Espaces publics de proximité, économie solidaire et démocratisation de l'économie », Revue Hermès, n³6, pp. 137-147

Fraisse, L. et Eme, B. 2005, « La gouvernance locale de la diversification des modes d'accueil : un enjeu de cohésion sociale », Recherches et Prévisions, CNAF, $n^{\circ} 80$, juin.

Guigue, B., 2000, «Qu'est-ce que l'économie solidaire? », Problèmes économiques, $\mathrm{n}^{\circ} 2677$.

Habermas, J., 1997, Droit et démocratie, Paris : Gallimard.

Habermas, J., 1986, L'Espace public, Paris : Payot.

Laville J-L., 2003, « Démocratie et économie : éléments pour approche sociologique », Hermès 36, pp. 185-194

Laville, J.-L., 1999, « La démocratisation du travail par l'économie solidaire », Roanne, communication aux Rencontre nationales de l'économie solidaire.

Laville, J.-L., 2000, L'Economie solidaire. Une perspective internationale, Paris : Desclée de Brouwer, réédition.

Laville, J-L., 1999, « Économie solidaire et tiers secteur », Transversales, nº 57, mai-juin.

Laville, J-L. et Nyssens,M., 2001, Les Services sociaux, entre associations, État et marché, Paris : La Découverte, Mauss, Crida, pp. 233-255.

Rosanvallon, P., 1998, « Le nouveau travail de la représentation », Esprit, février. 